\title{
The evolution of cooperation is affected by the persistence of fitness effects, the neighborhood size and their interaction
}

\author{
Eli Thompson ${ }^{\mathrm{a}}$, Jasmine Everett ${ }^{\mathrm{b}}$, Jonathan T. Rowellc, Jan Rychtář ${ }^{\mathrm{c}}$ and Olav Rueppelld \\ ${ }^{a}$ Department of Mathematics, Miami University, Oxford, $\mathrm{OH}, \mathrm{USA}$; ${ }^{\mathrm{b}}$ Department of Mathematics and Computer \\ Science, Bennett College, Greensboro, NC, USA; 'Department of Mathematics and Statistics, The University of \\ North Carolina at Greensboro, Greensboro, NC, USA; 'Department of Biology, The University of North Carolina \\ at Greensboro, Greensboro, NC, USA
}

\begin{abstract}
Evolutionary game theory and the Prisoner's Dilemma (PD) Game in particular have been used to study the evolution of cooperation. We consider a population of asexually reproducing, age-structured individuals in a two-dimensional square-lattice structure. The individuals employ fixed cooperative or defecting strategy towards their neighbours in repeating interactions to accumulate reproductive fitness. We focus on the effects of the persistence of past interactions and interactive neighbourhood size on the evolution of cooperation. We show that larger neighbourhood sizes are generally detrimental to cooperation and that the persistence of fitness effects decreases the likelihood of the evolution of cooperation in small neighbourhoods. However, for larger neighbourhood sizes the persistence effect is reversed. Thus, our study corroborates earlier studies that population structure increases the evolutionary potential for cooperative behaviour in a PD paradigm. This finding may explain the heterogeneity of previous results on the effect of neighbourhood size and cautions that the persistence of fitness outcomes needs to be considered in analyses of the evolution of cooperative behaviour. The persistence of fitness outcomes of pairwise interactions may vary dramatically in biological and social systems and could have profound effects on the evolution of cooperation in various contexts.
\end{abstract}

\section{ARTICLE HISTORY}

Received 15 July 2015

Accepted 22 August 2015

\section{KEYWORDS}

Social evolution; altruism; persistence; game theory; population viscosity

\section{Introduction}

Game-theoretical models have been key for analysing which conditions favour the evolution of cooperation, an important precedent for sociality, in recent decades (Broom \& Rychtár, 2013; Nowak, 2006). An especially relevant game for studying cooperation is the classic Prisoner's Dilemma (PD). Within this two-player game, each individual chooses one strategy: cooperation or defection. Cooperators help others but pay a cost to do so, while defectors neither provide benefits nor pay costs but do benefit from cooperators. While mutual cooperation would result in the highest total payoff for all individuals, an individual's own 
best strategy in the simple case is always to defect, regardless of the opponent's strategy. Thus, the evolution of cooperation is hard to explain without applying additional conditions.

One mechanism that favours the evolution of cooperation is spatial structure within the population, which allows individuals to play the PD game with a subset of the entire population, rather than with every individual. Spatial structure within an iterated PD game has proven to allow cooperation to invade an all-defecting population, which is not possible without the spatial structure (Chiong et al., 2007; Nakamaru et al., 1997; Nowak \& May, 1992; Pan et al., 2013; Wang et al., 2015, 2013, 2013, 2015). Such structure also promotes cooperation within a non-iterated PD game by allowing local clusters of mutually reinforcing cooperators to overcome the cost that individual cooperators pay compared to defectors. Specifically, it has been found that cooperation can persist within a spatial structure if the benefit of the altruistic act, $b$, divided by the cost, $c$, exceeds the average number of neighbours' (Ohtsuki et al., 2006); in particular, if $b>c$.

Another mechanism that can promote cooperation within a population is allowing PD games to have a long-lasting effect (persistence) on an individual's reproductive fitness. In a one-dimensional spatial structure, persistence is actually necessary to sustain cooperation (Gelimson et al., 2013). Furthermore, in ordered lattice spatial structures, persistence hinders the spread of defectors along the lattice, promoting cooperation in an iterated PD model (Alonso-Sanz, 2009a, 2009b). Note that persistence is different from a memory that allows individuals to adjust behaviour based on past interactions, or reputation of their interactants; although the memory can also promote the evolution of cooperation (Horvath et al., 2012; Moreira et al., 2013).

Life history describes the schedule and duration of key events in an organism's lifetime, particularly the age-specific survival and reproductive schedule. Different life history structures have proven to affect the evolution of many traits, such as juvenile dispersal strategies, as well as helping behaviour and altruism (Lehmann \& Rousset, 2010; Ronce et al., 2000; Wang, Zhu, \& Arenzon, 2012). Many studies have also examined age structure within various spatial structures in iterated PD models, examining the spread of cooperation in that regard (Irwin \& Taylor, 2001; Taylor \& Irwin, 2000). The timing of life history events also affects the evolution of cooperation within pure strategy spatial PD games. Individuals with long post-reproductive life spans (humans, for example) are more likely to cooperate, and an increased ratio of post-reproductive to pre-reproductive lifespan also favours the spread of cooperation in a population (Ross et al., 2015).

We aim to unite the mechanisms described above for the evolution of cooperation into one model to study their interaction and better understand how cooperation can evolve within a biological population with strictly vertical strategy transmission. Our model incorporates spatial structure in the form of a two-dimensional square-lattice. Individuals play the PD game with a number of potential neighbours set by varying sizes of neighbourhoods within the lattice structure. The population is age-structured: individuals move through pre-reproductive, reproductive and post-reproductive stages, and can also prematurely die within these stages at rates that we can manipulate. Our model allows both current and past interactions to determine the current reproductive fitness of an individual, and we can vary the degree of influence of past interactions. Fitness effects accrue incrementally throughout an individual's entire life but the persistence of past interaction effects varies (Gelimson et al., 2013). 

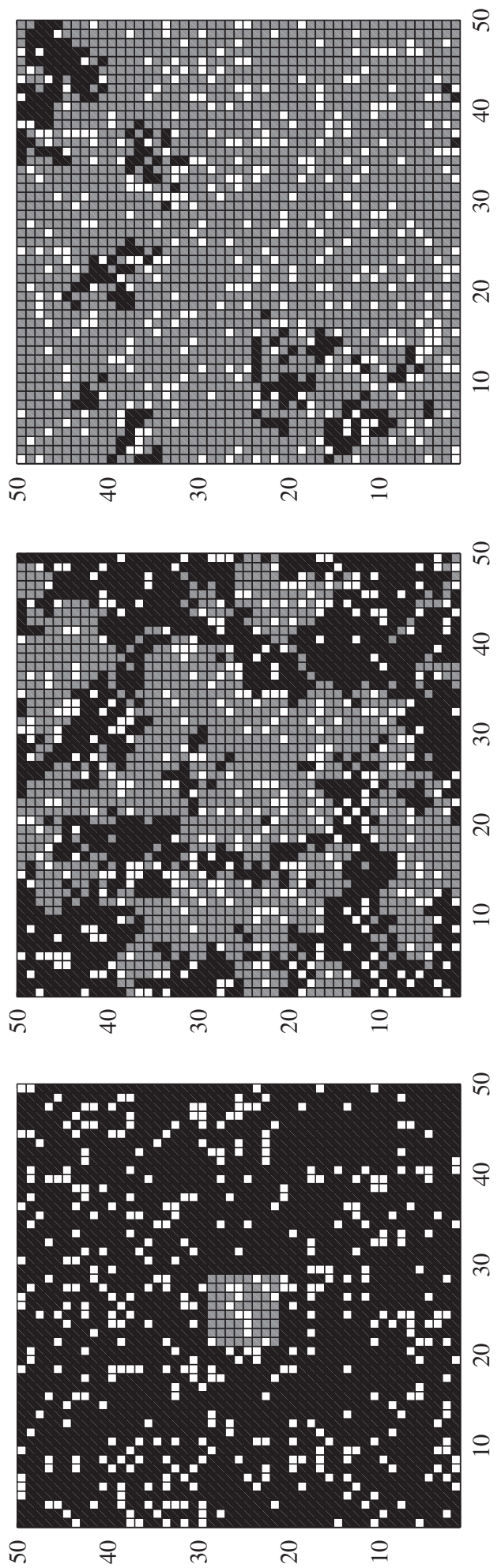

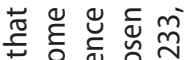

जิ 원

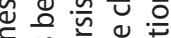

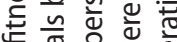

늠흥 원

근 흥 워

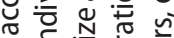

으

눙응 꿍

흥응 은 능 응

.

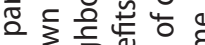

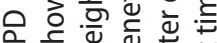

o

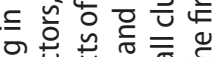

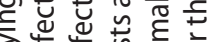
तิ

的吃

든

자을 뚱

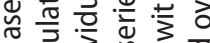

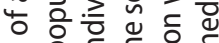

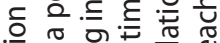

윰 $\cong$ 舟

吾

흥응

드응ㅎㅇ

즁

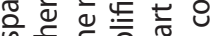

记 은

$\subseteq \sum$ ते

응

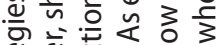

$\pm$

논

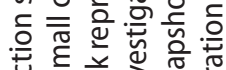

的离苋苋

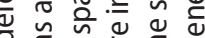

政

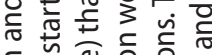

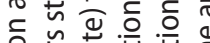

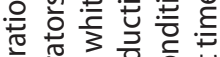

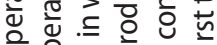

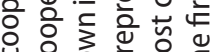

ช 일

ชั

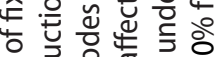

气 등 은

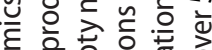

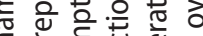

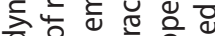

응 ॠ

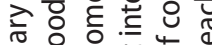

ठ등

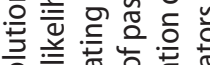

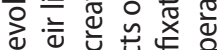

\%

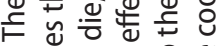

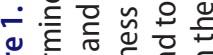

亏 诃

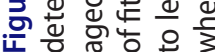


We focus on the effects and interaction of neighbourhood size and persistence of fitness outcomes. Our model results suggest that the persistence of past interactions has a negative effect on the evolution of cooperation from an initially small cooperative cluster, when the neighbourhood size is one (Moore Neighbourhood). However, increasing the neighbourhood size reverses this relationship, promoting cooperation with increased persistence of fitness effects. In general, a larger neighbourhood size is detrimental to the spread of cooperation. Our results also suggest that the speed of the spread of cooperation is influenced by an interaction between neighbourhood size and persistence of past fitness effects.

\section{Model}

To collect our simulation data, we built a spatially organized population model in MATLAB that extends the stochastic model put forth by Ross et al. (2015) by incorporating the persistence effects.

Individuals occupy nodes on the vertices of a 50 by 50 square lattice with periodic boundaries. This lattice is already large enough to avoid potential random effects caused by small population sizes, yet it is small enough for simulations to finish in reasonable time. We have tested larger lattice sizes and received qualitatively similar results. Every one of the 2500 nodes can interact in pairwise fashion with various numbers of neighbouring nodes defined by $N$; most simply with a neighbourhood size $N=1$, a Moore neighbourhood, the eight directly neighbouring nodes. The number of individuals in the population varies due to death events, and the average proportion of empty nodes after initialization is $1 / 6$, which is determined by the average life length of an individual. These interactions determine reproductive fitness of an individual based on the PD pay-off matrix

$$
\text { Cooperate Defect }\left(\begin{array}{ll}
1+b-c & 1 \\
1+b & 1
\end{array}\right)
$$

where $b, c>0$. We used $b=1.2$ and $c=0.2$ throughout this paper so that $b / c>4$ in order to satisfy the criterion for the evolution of cooperation from Ohtsuki et al. (2006). Small changes in $b$ and $c$ did not qualitatively change the outcomes. Each individual's strategy remains fixed throughout its life.

Our model updates stochastically. Each time step one node is chosen uniformly at random from the entire lattice and updated. If the chosen node is occupied, that individual progresses to the next life-history stage with a probability, $p_{x}$, where $d_{x}=1 / p_{x}$ is the expected duration of life-history stage $x$.

The model includes pre-reproductive, reproductive and post-reproductive life-history stages, with durations $d_{\text {pre }}, d_{\text {rep }}$ and $d_{\text {post }}$, respectively. The average expected life duration (in the absence of any premature mortality factor) $d_{\text {life }}$ is given by $d_{\text {pre }}+d_{\text {rep }}+d_{\text {post }}$. We fix $d_{\text {pre }}=1, d_{\text {rep }}=3$ and $d_{\text {post }}=1$ to approximately mimic life-history stage proportions in humans (Nichols et al., 2006). It should be noted that these lengths themselves affect the evolution of cooperation (Ross et al., 2015). A transition from the last life-history stage results in the 'natural' death of the individual, but our individuals can also experience premature death in the pre-reproductive and reproductive stages. The mortality rate for stage $x$, denoted $m_{x}$, is the probability that an individual dies before moving on to the next life 
stage. Thus, a probability that an individual in a pre-reproductive or reproductive stage $x$ dies during an update is given by

$$
m_{x}^{*}=1-\left(1-m_{x}\right)^{\left(1 / d_{x}\right)}
$$

Every individual in our population collects pay-offs from the games with neighbouring individuals. The pay-offs from individual interactions contribute to the cumulative fitness of the individual. The fitness of an individual occupying node $i$ is denoted $f_{i}$, which is presumed to be $f_{i}=1$ when an individual is born. Whenever the individual occupying node $i$ is updated, the cumulative fitness is updated by

$$
f_{i} \leftarrow \pi f_{i}+(1-\pi) \sum_{j \in \operatorname{Nbhd}(i)} P(i, j)
$$

where $0<\pi \leq 1$ corresponds to the persistence of the effects of past interactions on current fitness and $P(i, j)$ is the pay-off to an individual in $i$ from the interaction with an individual in $j$. The exact pay-off values come from the PD pay-off matrix (1).

If node $i$ is currently empty, i.e. the individual previously occupying the node has died, the surrounding neighbours are assessed to determine which one will reproduce and fill the empty node with an offspring of identical strategy. If none of the neighbours are of reproductive age, the chosen node remains empty. Otherwise, a reproductive neighbour $j$ is chosen with probability proportional to its fitness relative to the fitness of all other neighbours of reproductive age,

$$
\frac{f_{j} \cdot r_{j}}{\sum_{k \in \operatorname{Nbhd}(i)} f_{k} \cdot r_{k}}
$$

where

$$
r_{j}= \begin{cases}1 & \text { if } j \text { is at the reproductive stage } \\ 0 & \text { otherwise }\end{cases}
$$

is an indicator of being in the reproductive stage. A pre-reproductive individual is placed in the empty node $i$ with the same strategy as the individual selected for the reproduction and with fitness $f_{i}=1$.

We consider one generation to be equal to the average number of updates required to update every individual on the lattice from birth to death. Therefore, one generation is equivalent to $50^{2} d_{\text {life }}=12,500$ individual updates. Each simulation was performed for a maximum of $G_{\max }=1000$ generation, starting with an initial population comprised of mostly defectors and a cluster of cooperators representing no more than $2.5 \%$ of the population. The initial age structure of the population always reflected the relative lengths of the life-history stages (Ross et al., 2015). Thus, initially $1 / 6$ of nodes were empty, $1 / 6$ contained pre-reproductive, $1 / 2$ reproductive and $1 / 6$ post-reproductive individuals.

An example of a specific simulation run is shown in Figure 1.

To examine the effects of persistence $(\pi)$, we ran 1000 repetitions varying $\pi$ from 0.1 to 1 in increments of 0.1 . This method was repeated for neighbourhood sizes, $N \in\{1,2,3,4\}$. 


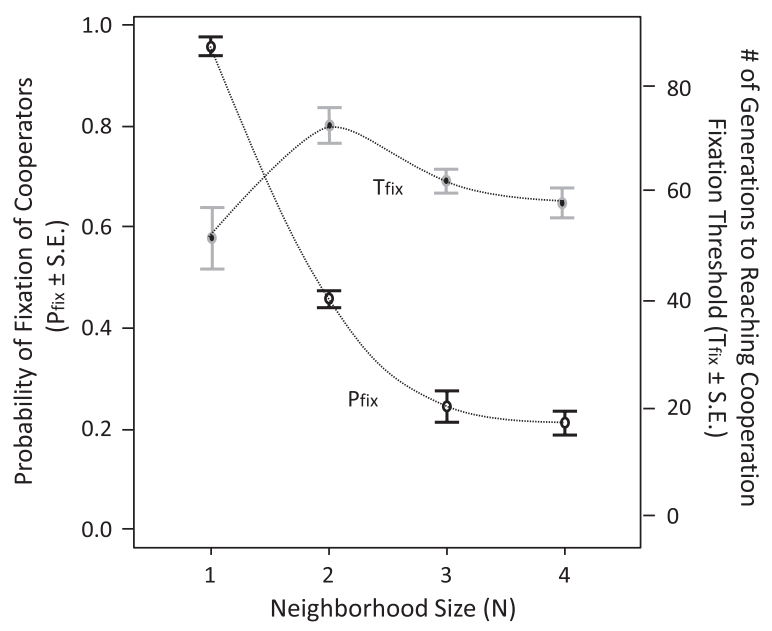

Figure 2. Across all levels of persistence of fitness effects, the probability of cooperator fixation decreased strongly with increasing neighbourhood size. This well-known effect is due to the loss of spatial separation between cooperators and defectors that breaks down cooperative clusters. The number of generations required to reach the threshold of cooperator fixation is influenced by the fitness differential between cooperators and defectors and by the spatial range of reproduction. Consequently, it shows a more complicated relationship with neighbourhood size, with a maximum at $N=2, m_{\text {pre }}=0.1, m_{\text {rep }}=0.1$, $\pi \in\{0.1,0.2, \ldots, 0.9,1\}$.
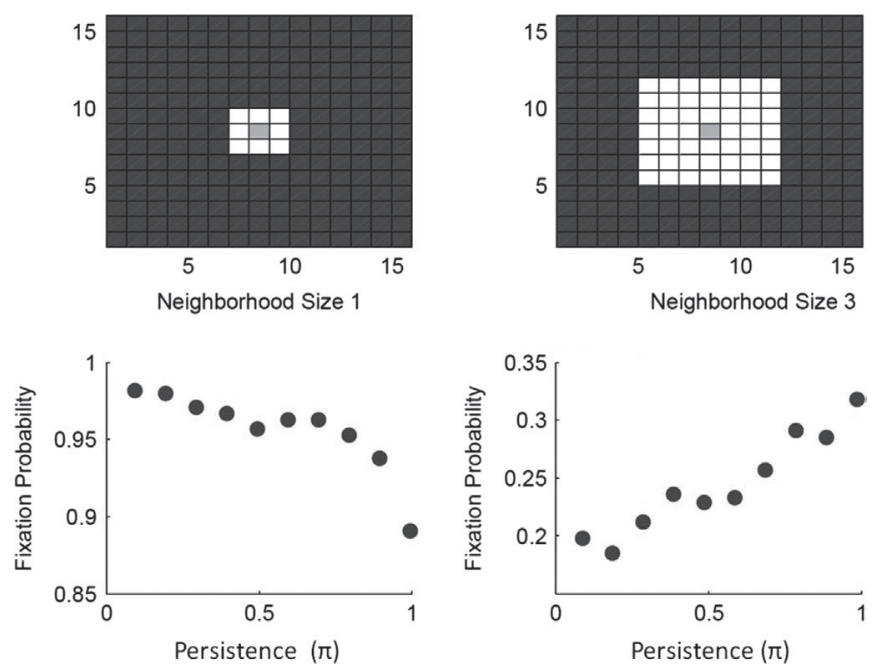

Figure 3. The size of the neighbourhood for pairwise interactions $(N)$ determined the effect of the persistence of fitness outcomes of past interactions on the probability of cooperator fixation. While persistence impacted the evolution of cooperation negatively in a Moore neighbourhood, all studied larger neighbourhood sizes $(N=2,3,4)$ showed a positive relation between persistence and the probability for cooperation to become fixed in the population. $m_{\text {pre }}=0.1, m_{\text {rep }}=0.1$.

Premature mortality is one stochastic mechanism that not only diminishes the persistence of fitness effects, but also creates more reproductive opportunities. To examine the effects of pre-reproductive mortality, $m_{\text {pre }}$, we varied it between 0 and 0.5 in increments of 0.1 , while 


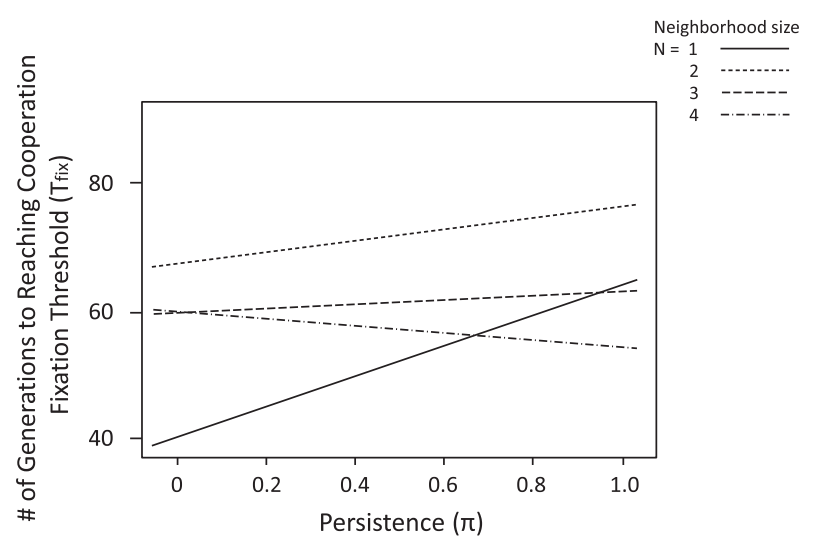

Figure 4. The size of the neighbourhood for pairwise interactions $(N)$ determined the effect of the persistence of fitness outcomes of past interactions on the number of generations required to reach a pre-determined threshold of cooperator fixation. While persistence significantly slowed the spread of cooperation in a Moore neighbourhood, this effect was decreased in larger neighbourhood sizes. $m_{\text {pre }}=0.1, m_{\text {rep }}=0.1$.
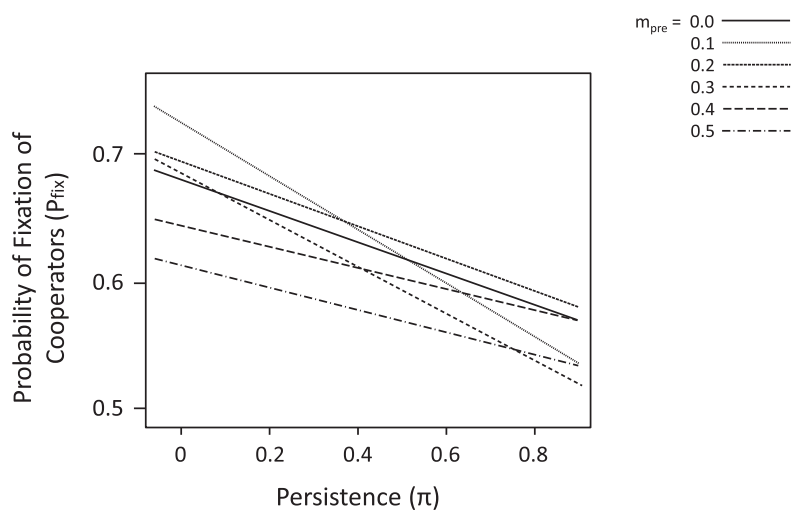

Figure 5. For $N=1$, increasing the persistence decreases the fixation probability for cooperators across all values of $m_{\text {pre }}\left(m_{\text {rep }}=0.1\right)$.

simultaneously varying the persistence, $\pi$, from 0.1 to 1 with $N=1$, for 1000 independent replicate simulations.

For each simulation, we recorded the final proportion of cooperators in the population, and how many generations were required to reach cooperator fixation, if it occurred. Based on the preliminary trials, we determined that once cooperators reached $10 \%$ of the total population, cooperation was established enough to inevitably fixate. For this reason, we terminated simulations before $G_{\max }$ when $10 \%$ cooperation (cooperator fixation) or $0 \%$ cooperation (defector fixation) was reached. We then calculated the probability of cooperator fixation for a given scenario as the percentage of replicate simulations in which cooperation exceeded the $10 \%$ threshold, $P_{\text {fix }}$. In addition, we calculated the fixation time, $T_{\text {fix }}$, as the average number of generations required to reach the $10 \%$ threshold. The effect of each investigated parameter on these outcomes was evaluated separately by linear regression in SPSS (IBM), reporting unstandardized coefficients with their standard error and adjusted $r^{2}$ values. 

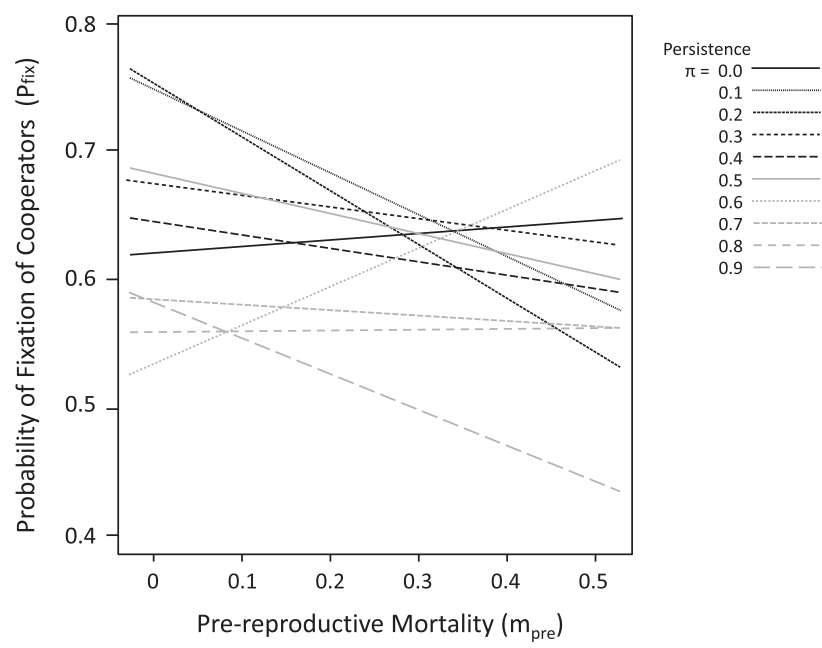

Figure 6 . For $N=1$, the effect of pre-reproductive mortality varies with the fitness persistence level $\left(m_{\text {rep }}=0.1\right)$.

\section{Results}

Across all persistence levels, the size of the neighbourhood decreased the probability of the evolution of cooperation but its effect on the speed of cooperator fixation was more complicated (Figure 2).

The initial model, employing a Moore neighbourhood $(N=1)$, indicated a negative effect of fitness persistence on the evolution of cooperation (Figure 3, left panel; $B=-0.07 \pm 0.02$, $\left.r^{2}=0.66, n=9, p=0.003\right)$. However, the effect of fitness persistence was opposite for the three other neighbourhood sizes, increasing the probability of cooperator fixation. For a neighbourhood size of $N=2, B=0.06 \pm 0.02, r^{2}=0.51, n=9, p=0.012$, for $N=3$, $B=0.14 \pm 0.01, r^{2}=0.91, n=9, p<0.001$ and for $N=4, B=0.09 \pm 0.02, r^{2}=0.61, n=9$, $p=0.005$ (Figure 3, right panel).

Across all cases that led to the fixation of cooperation, the time required for the $10 \%$ threshold to be reached, $T_{\text {fix }}$, was significantly affected by neither persistence nor the neighbourhood size, $N\left(\pi: B=7.8 \pm 4.9, p=0.122 ; N: B=1.0 \pm 1.3, p=0.449, r^{2}=0.03, n=40\right.$, $p=0.227$ ). However, analysed separately, the original Moore neighbourhood model indicated a strong positive effect of persistence on $T_{f i x}\left(B=24.2 \pm 3.5 r^{2}=0.84, n=9, p<0.001\right)$. This effect turned negative with increasing neighbourhood size (Figure 4), although none of the remaining analysis indicated significance $\left(N=2, B=8.6 \pm 4.3, r^{2}=0.25\right.$, $n=9, p=0.078$, for $N=3, B=3.5 \pm 3.2, r^{2}=0.02, n=9, p=0.304$ and for $N=4$, $\left.B=-5.2 \pm 4.2, r^{2}=0.05, n=9, p=0.260\right)$. Across all scenarios, $P_{\text {fix }}$ and $T_{\text {fix }}$ were negatively correlated $(r=-0.41, n=40, p=0.008)$.

Simultaneous evaluation of premature mortality and the persistence of fitness effects in a Moore neighbourhood indicated an overall negative effect of both $\left(m_{\text {pre }}: B=-0.11 \pm 0.05\right.$, $\left.p=0.035 ; w: B=-0.14 \pm 0.03, p<0.001 ; r^{2}=0.30, n=60, p<0.001\right)$. While the negative effect of persistence was consistent across all levels of mortality (Figure 5), the effects of premature mortality varied across the levels of persistence (Figure 6). In these simulations, the time required for cooperator fixation, $C_{\text {gen }}$, was decreased by premature 
mortality and increased by the persistence of fitness effects ( $m_{\text {pre }}: B=-13.8 \pm 4.0, p=0.001$; $\left.w: B=26.7 \pm 2.4, p<0.001 ; r^{2}=0.70, n=60, p<0.001\right)$. Across all scenarios, $P_{\text {fix }}$ and $T_{\text {fix }}$ were significantly correlated $(r=-0.44, n=60, p<0.001)$.

\section{Discussion}

Our results show that the persistence of fitness effects of past interactions can impair the evolution of cooperation under certain circumstance. We also show that a larger neighbourhood size decreases the probability for cooperation to evolve in general. However, neighbourhood size and persistence of fitness effects interact. In larger neighbourhoods, the effect of persistence reverses, favouring the evolution of cooperation. Contrary to our prediction, premature mortality did not exhibit a clear effect on the evolution of cooperation.

Our results indicate that persisting fitness effects of past interactions is detrimental to the spread of cooperation in a neighbourhood size of one. In this case, the speed at which cooperation spreads through the population (when it does) is also slowed by increasing the persistence of fitness outcomes of past interactions. In larger neighbourhood sizes, however, increased persistence increases the probability of the evolution of cooperation and the negative effect of persistence on the speed of cooperator fixation also weakens with increasing neighbourhood size accordingly.

For $1 \leq N \leq 4$, increasing $N$ decreases the fixation probability of cooperation. This result has been well studied (Nowak, 2006; Ohtsuki et al., 2006; Ross et al., 2013). Larger neighbourhood size blurs the boundary between cooperators and defectors, allowing more defectors to benefit from cooperators in the cluster. As the neighbourhood size increases, the population approaches being well mixed, which is detrimental to cooperation. Increasing neighbourhood size also allows the possibility of defectors reproducing into the interior of the cluster, where defector fitness is especially high. The added benefit to the defectors near the boundaries overcomes the benefit the cooperators receive from interacting with more cooperators, decreasing the probability that cooperators can establish themselves in the population.

The fixation probability is strongly influenced by the initial generations when the small cooperative starting cluster either grows or dies out. This critical initiation phase in our model may explain why our results on fitness persistence in a Moore neighbourhood impeded the evolution of cooperation in contrast to a previous study Gelimson et al. (2013). A similar result is mentioned briefly in Alonso-Sanz (2009a, Figure 4), in which the cooperative population initially plummets, and the persistence does not seem to help cooperation to establish itself. If $N=1$, only reproduction occurring directly on the boundary of the cooperating cluster influences the proportion of cooperators and defectors in the population. Increased persistence of fitness effects functionally increases the neighbourhood size by extending it to past neighbours that may have died in the time between their interaction with the focal individual and reproduction of the focal individual. This effect benefits the defectors and hurts the cooperators at the boundaries, allowing the defectors to break down the initially small cooperative cluster. This effect impacts the simulation less with physically larger neighbourhood sizes and thus a positive relation between persistence and cooperator evolution is observed, as in other studies (Alonso-Sanz, 2009b; Gelimson et al., 2013).

Similarly to the fixation probability, the number of generations required to reach the threshold for cooperator fixation is affected by an interaction between neighbourhood size 
and persistence of fitness effects: In small physical neighbourhoods the effect of integrating neighbourhoods over time decreases the speed at which cooperation spreads through the population by effectively increasing the functional neighbourhood size. Our model leads to a rapid spread of cooperation when a Moore neighbourhood is employed because the tight cluster strongly favours cooperation. In this case, persistent fitness effects slow the spread of cooperation at the boundary of the cooperative cluster by accounting for past interactions. Employing larger neighbourhood sizes allows individuals to access interior nodes across the boundary and decreases the benefit of the cooperative cluster at the boundary. The former makes persistence beneficial for cooperation because it prevents intrusion of defectors into the cooperative cluster. The latter decreases the negative effect of persistence on the speed of the spread of the cooperative cluster. Thus, both factors are important for explaining the interaction between neighbourhood size and the persistence of fitness effects.

Pre-reproductive mortality is in many species a considerable selective factor (Finch, 1994). We introduced premature mortality during the pre-reproductive phase, as a natural variable that eliminates individuals irrespective of fitness accumulation and can stochastically create more reproductive opportunities in the population. The negative effect of fitness persistence on the evolution of cooperators in a Moore neighbourhood model was confirmed by this second set of simulations. Moreover, different levels of pre-reproductive mortality seem not to affect this relation in a systematic fashion. Pre-reproductive mortality decreased the probability of cooperator fixation overall, although it did not vary between cooperators and defectors. The effect was observed in most but not all levels of fitness persistence, no clear pattern emerged on the interaction between fitness persistence and pre-reproductive mortality. The overall effect occurs most likely by increasing the stochasticity of the system through increased mortality, which can lead to the extinction of the initially small cooperative cluster even in scenarios when cooperation would be favoured in the long term. This interpretation is supported by the result that pre-reproductive mortality decreases the number of generations required for the fixation of cooperators.

Our model seeks to emulate populations of biological species with a natural life history and strictly vertical inheritance of behavioural strategies (Ross et al., 2013, 2015). However, we make several assumptions regarding the persistence of fitness effects (fitness collection) and reproduction that might affect the generality of our results. One assumption is that fitness is cumulative and not averaged over the number of neighbours an individual has upon fitness collection. Thus, individuals with more live neighbours are more fit than individuals with empty neighbouring nodes. While this may hold true in some biological systems (Carter \& Wilkinson, 2013) in other species available space in adjacent territory may increase personal fitness by decreasing intra-specific competition. The way, we evaluate fitness could affect the reproductive behaviour of individuals, but it is unlikely to bias our results because mortality is independent of strategy or fitness and thus cooperators and defectors encounter empty nodes at similar rates. The simplification that fitness affects only reproduction but not mortality is not biologically realistic. Fitness effects on survival have been previously incorporated into the analysis of social evolution in a kin-selection framework (Pen, 2000; Taylor \& Irwin, 2000), but it remains to be elucidated how fitness-dependent mortality alters the evolution of cooperation in a strict PD paradigm. Another promising extension of our age-structured model is the incorporation of age-dependent costs and benefits in the PD game (Wang et al., 2012), which also can be biologically justified (Lee, 2003). 


\section{Acknowledgements}

The authors would like to thank Quinn Morris and Liz Bergen, who worked for the UNCG REU as graduate student assistants during summer 2014.

\section{Disclosure statement}

No potential conflict of interest was reported by the authors.

\section{Funding}

Eli Thompson and Jasmine Everett were undergraduate student participants of the UNCG REU program in Mathematical Biology during summer 2014, supported by the National Science Foundation (grant DMS-1359187). The work was additionally supported by the National Institutes of Health (NIA grant R21AG046837).

\section{References}

Alonso-Sanz, R. (2009a). Memory versus spatial disorder in the support of cooperation. Biosystems, 97, 90-102.

Alonso-Sanz, R. (2009b). Spatial order prevails over memory in boosting cooperation in the iterated prisoner's dilemma. Chaos: An Interdisciplinary. Journal of Nonlinear Science, 19(2), 023102.

Broom, M., \& Rychtáŕ, J. (2013). Game-theoretical models in biology. Boca Raton, FL: CRC Press.

Carter, G. G., \& Wilkinson, G. S. (2013). Food sharing in vampire bats: Reciprocal help predicts donations more than relatedness or harassment. Proceedings of the Royal Society of London B: Biological Sciences, 280, 20122573. Retrieved from http://dx.doi.org/10.1098/rspb.2012.2573

Chiong, R., Dhakal, S., \& Jankovic, L. (2007). Effects of neighbourhood structure on evolution of cooperation in $n$-player iterated prisoners dilemma. In H. Yin, P. Tino, E. Corchado, W. Byrne, X. Yao (Eds.), Intelligent Data Engineering and Automated Learning-IDEAL 2007 (pp. 950-959). Berlin, Heidelberg: Springer-Verlag.

Finch, C. E. (1994). Longevity, senescence, and the genome. Chicago: University of Chicago Press.

Gelimson, A., Cremer, J., \& Frey, E. (2013). Mobility, fitness collection, and the breakdown of cooperation. Physical Review E, 87, 042711.

Horvath, G., Kovárík, J., \& Mengel, F. (2012). Limited memory can be beneficial for the evolution of cooperation. Journal of Theoretical Biology, 300, 193-205.

Irwin, A. J., \& Taylor, P. D. (2001). Evolution of altruism in stepping-stone populations with overlapping generations. Theoretical Population Biology, 60, 315-325.

Lee, R. D. (2003). Rethinking the evolutionary theory of aging: Transfers, not births, shape senescence in social species. Proceedings of the National Academy of Sciences, 100, 9637-9642.

Lehmann, L., \& Rousset, F. (2010). How life history and demography promote or inhibit the evolution of helping behaviours. Philosophical Transactions of the Royal Society B: Biological Sciences, 365, 2599-2617.

Moreira, J., Vukov, J., Sousa, C., Santos, F. C., d'Almeida, A. F., Santos, M. D., \& Pacheco, J. M. (2013). Individual memory and the emergence of cooperation. Animal Behaviour, 85, 233-239.

Nakamaru, M., Matsuda, H., \& Iwasa, Y. (1997). The evolution of cooperation in a lattice-structured population. Journal of Theoretical Biology, 184, 65-81.

Nichols, H. B., Trentham-Dietz, A., Hampton, J. M., Titus-Ernstoff, L., Egan, K. M., Willett, W. C., \& Newcomb, P. A. (2006). From menarche to menopause: Trends among us women born from 1912 to 1969. American Journal of Epidemiology, 164, 1003-1011.

Nowak, M. A. (2006). Five rules for the evolution of cooperation. Science, 314, 1560-1563.

Nowak, M. A., \& May, R. M. (1992). Evolutionary games and spatial chaos. Nature, 359, 826-829.

Ohtsuki, H., Hauert, C., Lieberman, E., \& Nowak, M. A. (2006). A simple rule for the evolution of cooperation on graphs and social networks. Nature, 441, 502-505. 
Pan, Q., Shi, S., Zhang, Y., \& He, M. (2013). Cooperation in spatial prisoners dilemma game with delayed decisions. Chaos, Solitons \& Fractals, 56, 166-174.

Pen, I. (2000). Reproductive effort in viscous populations. Evolution, 54, 293-297.

Ronce, O., Gandon, S., \& Rousset, F. (2000). Kin selection and natal dispersal in an age-structured population. Theoretical Population Biology, 58, 143-159.

Ross, C., Rueppell, O., \& Rychtáŕ, J. (2013). A spatially organized population model to study the evolution of cooperation in species with discrete life-history stages. In Rychtár., Gupta, Shivaji, Chhetri, (Eds.), Topics from the 8th Annual UNCG Regional Mathematics and Statistics Conference (pp. 147-154). New York, NY: Springer.

Ross, C., Rychtář, J., \& Rueppell, O. (2015). A structured population model suggests that long life and post-reproductive lifespan promote the evolution of cooperation. Journal of Theoretical Biology, 369, 85-94.

Taylor, P. D., \& Irwin, A. J. (2000). Overlapping generations can promote altruistic behavior. Evolution, $54,1135-1141$.

Wang, Z., Andrews, M. A., Wu, Z. X., Wang, L., \& Bauch, C. T. (2015). Coupled disease-behavior dynamics on complex networks: A review. Physics of Life Reviews. doi:10.1016/j.plrev.2015.07.006

Wang, Z., Kokubo, S., Tanimoto, J., Fukuda, E., \& Shigaki, K. (2013). Insight into the so-called spatial reciprocity. Physical Review E, 88, 042145.

Wang, Z., Szolnoki, A., \& Perc, M. (2013). Optimal interdependence between networks for the evolution of cooperation. Scientific Reports, 3, 2470. doi: 10.1038/srep02470

Wang, Z., Wang, L., Szolnoki, A., \& Perc, M. (2015). Evolutionary games on multilayer networks: A colloquium. The European Physical Journal B, 88(5), 1-15.

Wang, Z., Zhu, X., \& Arenzon, J. J. (2012). Cooperation and age structure in spatial games. Physical Review E, 85, 011149. 